\title{
Fixed Point Results and E. A Property in Dislocated and Dislocated Quasi- metric Spaces
}

\author{
Kastriot Zoto $^{1, *}$, Arben Isufati ${ }^{2}$, Panda Sumati Kumari ${ }^{2}$ \\ ${ }^{1}$ Department of Mathematics and Computer Sciences, Faculty of Natural Sciences, University of Gjirokastra, Albania \\ ${ }^{2}$ Department of Mathematics, KL University, Green Fields, Andhra Pradesh, India \\ *Corresponding author: zotokastriot@yahoo.com
}

Received December 24, 2014; Revised January 26, 2015; Accepted March 01, 2015

\begin{abstract}
We prove several fixed points theorems for weakly compatible selfmappings on a dislocated and dislocated quasi-metric space, which satisfy E. A Like and common E. A. Like property, satisfying liner type of contractive condition.
\end{abstract}

Keywords: dislocated metric, dislocated quasi-metric, common E.A Like property, contraction, fixed point

Cite This Article: Kastriot Zoto, Arben Isufati, and Panda Sumati Kumari, "Fixed Point Results and E. A Property in Dislocated and Dislocated Quasi- metric Spaces." Turkish Journal of Analysis and Number Theory, vol. 3, no. 1 (2015): 24-29. doi: 10.12691/tjant-3-1-6.

\section{Introduction}

Fixed point theory as a part of functional analysis has many applications in diverse fields of sciences as electronic engineering, computer sciences, game theory, biology and physics. In these recent years the extension of metric fixed point theory to generalized metrics such as quasi-metric, b-metric, partial metric, dislocated metric, dislocated quasi-metric, b-dislocated quasi-metric has received much attention (see, for instance [2-20]. P. Hitzler and A. K. Seda [10] gave a modified version of the Banach contraction principle in dislocated metric spaces. Later F. M. Zeyada et al generalized that in dislocated quasi-metric space. Subsequently, several authors have studied the problem of existence and uniqueness of a fixed point for mappings satisfying different contractive conditions.

The purpose of this paper is to study the existence of a common fixed point for one and two pairs of mappings wich satisfy the E. A. Like and the common E. A. Like property in the framework of dislocated and dislocated quasi-metric space, extending some existing results.

\section{Preliminaries}

Definition 2.1 [10] Let $X$ be a non-empty and let $d: X \times X \rightarrow \mathbb{R}^{+}$be a function, called a distance function if for all $x, y, z \in X$, satisfies:

$$
\begin{gathered}
d_{1}: d(x, x)=0 \\
d_{2}: d(x, y)=d(y, x)=0 \Rightarrow x=y \\
d_{3}: d(x, y)=d(y, x) \\
d_{4}: d(x, y) \leq d(x, z)+d(z, y) .
\end{gathered}
$$

If $d$ satisfies the condition $d_{1}-d_{4}$, then $d$ is called a metric on $X$. If $d$ satisfies conditions $d_{2}, d_{3}$ and $d_{4}$ it is called a dislocated metric (or simply $d$-metric). If $d$ satisfies only $d_{2}$ and $d_{4}$ then $d$ is called a dislocated quasi-metric (or simply dq-metric) on $X$. A nonempty set $X$ with dq-metric $d$, i. e., $(X, d)$ is called a dislocated quasi-metric space.

Definition 2.2 [10] A sequence $\left(x_{n}\right)$ in a $d$-metric space $(X, d)$ is called a Cauchy sequence if for all $\varepsilon>0$, $\exists n_{0} \in N$ such that $\forall m, n \geq n_{0}$, we have $d\left(x_{m}, x_{n}\right)<\varepsilon$.

Definition 2.3 [10] A sequence $\left(x_{n}\right)$ in $d$-metric space converges with respect to $d$, if there exists $x \in X$, such that $\lim _{n \rightarrow \infty} d\left(x_{n}, x\right)=0$.

In this case $x$ is called a d-limit of $\left(x_{n}\right)$ and we write $x_{n}$ $\rightarrow x$.

Definition 2.4 [10] A $d$-metric space $(X, d)$ is called complete if every Cauchy sequence in it is convergent with respect to $d$.

Definition 2.5 [10] Let $(X, d)$ be a d-metric space. A mapping $T: X \rightarrow X$ is called contraction if there exists a number $0 \leq \lambda<1$ such that $d(T x, T y) \leq \lambda d(x, y)$ for all $x, y \in X$.

Lemma $2.6[10]$ Let $(X, d)$ be a d-metric space. If $T: X \rightarrow X$ is a contraction function, then $T^{n}\left(x_{0}\right)$ is a Cauchy sequence for each $x_{0} \in X$.

Lemma 2.7 [10] Limits in a d-metric space are unique.

Theorem 2.8 [10] Let $(X, d)$ be complete d-metric space and let $T: X \rightarrow X$ be a contraction mapping then, $T$ has a unique fixed point. 
Definition 2.9 [5] Let $F$ and $S$ be mappings from a dislocated metric space $(X, d)$ into itself. Then, $F$ and $S$ are said to be weakly compatible if they commute at their coincidence point; that is $F x=S x$ for some $x \in X$ implies $S F x=F S x$.

The definitions of K. Wadhwa et al [21] can be adopt in dislocated metric spaces as in following.

Definition 2.10 Let $S$ and $T$ be two self-mappings of a dislocated metric space $(X, d)$. We say that $F$ and $S$ satisfy the property E. A. if there exists a sequence $\left(x_{n}\right)$ such that

$$
\lim _{n \rightarrow \infty} S x_{n}=\lim _{n \rightarrow \infty} T x_{n}=t \text { for some } t \in X .
$$

Definition 2.11 Let $S, T, F$ and $G$ are self-mappings of a dislocated metric space $(X, d)$. The pairs $(S, F)$ and $(T, G)$ satisfy Common E. A. property, if there exists two sequences $\left(x_{n}\right)$ and $\left(y_{n}\right)$ in $X$ such that

$$
\begin{aligned}
& \lim _{n \rightarrow \infty} S x_{n}=\lim _{n \rightarrow \infty} F x_{n}=\lim _{n \rightarrow \infty} G y_{n}=\lim _{n \rightarrow \infty} T y_{n}=t \\
& \text { for some } t \in X .
\end{aligned}
$$

Definition 2.12 Let $S$ and $T$ be two self-mappings of a dislocated metric space $(X, d)$. We say that $S$ and $T$ satisfy the E. A. Like property if there exists a sequence $\left(x_{n}\right)$ such that

$$
\begin{aligned}
& \lim _{n \rightarrow \infty} S x_{n}=\lim _{n \rightarrow \infty} T x_{n}=t \\
& \text { for some } t \in S(X) \text { or } t \in T(X), \\
& \text { i. e. } t \in S(X) \cup T(X) .
\end{aligned}
$$

Definition 2.13 Let $S, T, F$ and $G$ are self-mappings of a dislocated metric space $(X, d)$. The pairs $(S, F)$ and $(T, G)$ satisfy Common E. A. Like property, if there exists two sequences $\left(x_{n}\right)$ and $\left(y_{n}\right)$ in $X$ such that

$$
\lim _{n \rightarrow \infty} S x_{n}=\lim _{n \rightarrow \infty} F x_{n}=\lim _{n \rightarrow \infty} G y_{n}=\lim _{n \rightarrow \infty} T y_{n}=t
$$

where $t \in F(X) \cap G(X)$ or $t \in S(X) \cap T(X)$.

\section{Main Result}

Theorem 3.1 Let $(X, d)$ be a complete dislocated quasimetric space and $f, g: X \rightarrow X$ are two self maps satisfying the conditions:

$$
\begin{aligned}
d(f x, f y) \leq & \alpha d(f x, g y)+\beta d(g x, f y)+\gamma d(g x, g y) \\
& +\delta d(g y, f y)+\eta d(g x, f x)
\end{aligned}
$$

for all $x, y \in X$, where the constants $\alpha, \beta, \gamma, \delta, \eta$ are nonnegative and $0 \leq \alpha+\beta+\gamma+\delta+\eta<\frac{1}{2}$

(3.2) $f$ and $g$ satisfy E. A. Like Property

(3.3) $f$ and $g$ are weakly compatible

for all $x, y \in X$, and $0 \leq \alpha+\beta+\gamma+\delta+\eta<\frac{1}{2}$.
Then $f$ and $g$ have a unique common fixed point in $X$.

Proof: Since $f$ and $g$ satisfy the E. A. Like Property therefore exists a sequence $\left(x_{n}\right)$ in $X$ such that $\lim _{n \rightarrow \infty} f x_{n}=\lim _{n \rightarrow \infty} g x_{n}=t \in f(X)$ or $g(X)$.

Assume that $\lim _{n \rightarrow \infty} f x_{n}=t \in g(X)$. Therefore, $t=g u$ for some $u \in X$.

From condition (3.1) we have:

$$
\begin{aligned}
& d\left(f u, f x_{n}\right) \leq \alpha d\left(f u, g x_{n}\right)+\beta d\left(g u, f x_{n}\right) \\
& +\gamma d\left(g u, g x_{n}\right)+\delta d\left(g x_{n}, f x_{n}\right)+\eta d(g u, f u)
\end{aligned}
$$

Taking limit $n \rightarrow \infty$, we get

$$
\begin{aligned}
d(f u, t) & \leq \alpha d(f u, t)+\beta d(t, t)+\gamma d(t, t) \\
& +\delta d(t, t)+\eta d(t, f u) \\
& \leq(\alpha+\beta+\gamma+\delta) d(f u, t) \\
& +(\beta+\gamma+\delta+\eta) d(t, f u)
\end{aligned}
$$

As a result we have

$$
d(f u, t) \leq \frac{\beta+\gamma+\delta+\eta}{1-(\alpha+\beta+\gamma+\delta)} d(t, f u)
$$

Again from 3.1 have:

$$
\begin{aligned}
& d\left(f x_{n}, f u\right) \leq \alpha d\left(f x_{n}, g u\right)+\beta d\left(g x_{n}, f u\right) \\
& +\gamma d\left(g x_{n}, g u\right)+\delta d(g u, f u)+\eta d\left(g x_{n}, f x_{n}\right)
\end{aligned}
$$

Taking limit $n \rightarrow \infty$ we get

$$
\begin{aligned}
d(t, f u) & \leq \alpha d(t, t)+\beta d(t, f u)+\gamma d(t, t) \\
& +\delta d(t, f u)+\eta d(t, t) \\
& \leq(\alpha+\beta+\gamma+\delta+\eta) d(t, f u) \\
& +(\alpha+\gamma+\eta) d(f u, t)
\end{aligned}
$$

From this inequality have

$$
d(t, f u) \leq \frac{\alpha+\gamma+\eta}{1-(\alpha+\beta+\gamma+\delta+\eta)} d(f u, t) .
$$

Putting $c=\max \left\{c_{1}, c_{2}\right\}$ where $c_{1}=\frac{\beta+\gamma+\delta+\eta}{1-(\alpha+\beta+\gamma+\delta)}$ and $c_{2}=\frac{\alpha+\gamma+\eta}{1-(\alpha+\beta+\gamma+\delta+\eta)}$.

Using (1) and (2) we get $d(f u, t) \leq c^{2} d(f u, t)$, so $d(f u, t)=0$ since $0 \leq c<1$.

In the same way by (2) and (1) we get $d(t, f u)=0$

Therefore $d(t, f u)=d(f u, t)=0$ implies $f u=t$.

The weak compatibility of $f$ and $g$ implies that,

$$
f t=f g u=g f u=g t
$$

Let we show that $t$ is a common fixed point of $f$. According to the condition3.1, consider:

$$
\begin{aligned}
& d\left(f t, f x_{n}\right) \leq \alpha d\left(f t, g x_{n}\right)+\beta d\left(g t, f x_{n}\right) \\
& +\gamma d\left(g t, g x_{n}\right)+\delta d\left(g x_{n}, f x_{n}\right)+\eta d(g t, f t)
\end{aligned}
$$


Taking limit $n \rightarrow \infty$ get

$$
\begin{aligned}
d(f t, t) & \leq \alpha d(f t, t)+\beta d(f t, t)+\gamma d(f t, t) \\
& +\delta d(t, t)+\eta d(f t, f t) \\
& \leq(\alpha+\beta+\gamma+\delta+\eta) d(f t, t)+(\delta+\eta) d(t, f t)
\end{aligned}
$$

Further we have:

$$
d(f t, t) \leq \frac{\delta+\eta}{1-(\alpha+\beta+\gamma+\delta+\eta)} d(t, f t)
$$

In the same way from condition 3.1 have:

$$
d(t, f t) \leq \frac{\delta+\eta}{1-(\alpha+\beta+\gamma+\delta+\eta)} d(f t, t)
$$

Inequality (3) and (4) implies $d(f t, t)=0$, since $0 \leq \alpha+\beta+\gamma+\delta+\eta<\frac{1}{2}$.

In the same way by 3.1 we get $d(t, f t)=0$. So by property of dislocated quasi-metric comes $f t=t$, and as a result $f t=g t=t$. Hence, $t$ is a common fixed point of $f$ and $g$.

Uniqueness. Let $t \neq t_{1}$ be two common fixed points of the mappings $f$ and $g$. Then from (3.1) we have:

$$
\begin{aligned}
d\left(t, t_{1}\right) & =d\left(f t, f t_{1}\right) \\
\leq & \alpha d\left(f t, g t_{1}\right)+\beta d\left(g t, f t_{1}\right)+\gamma d\left(g t, g t_{1}\right) \\
& +\delta d\left(g t_{1}, g t_{1}\right)+\eta d(g t, g t) \\
& =\alpha d\left(t, t_{1}\right)+\beta d\left(t, t_{1}\right)+\gamma d\left(t, t_{1}\right) \\
& +\delta d\left(t_{1}, t_{1}\right)+\eta d(t, t) \\
& \leq(\alpha+\beta+\gamma+\delta+\eta) d\left(t, t_{1}\right)+(\delta+\eta) d\left(t_{1}, t\right)
\end{aligned}
$$

which implies $d\left(t, t_{1}\right) \leq \frac{\delta+\eta}{1-(\alpha+\beta+\gamma+\delta+\eta)} d\left(t_{1}, t\right)$. In the same as above have $d\left(t_{1}, t\right) \leq \frac{\delta+\eta}{1-(\alpha+\beta+\gamma+\delta+\eta)} d\left(t, t_{1}\right)$

These show that $d\left(t, t_{1}\right)=0$. In the same way $d\left(t_{1}, t\right)=0$. From $d\left(t, t_{1}\right)=d\left(t_{1}, t\right)=0$ we get, $t=t_{1}$.

Hence the proof is complete.

The following example illustrates our theorem.

Example 3.2 Let $X=R^{+}$. Define $d: X \times X \rightarrow[0, \infty)$ by

$$
d(x, y)=x+2 y \text { for all } x, y \in X .
$$

And define $f x=\frac{1}{5} x ; g x=\frac{1}{4} x$ for all $x \in X$, and for the sequence $x_{n}=\frac{1}{n}, n \in N$, have

$$
\lim _{n \rightarrow \infty} f x_{n}=\lim _{n \rightarrow \infty} g x_{n}=0 \in f(X) \cup g(X) .
$$

We observe that $\left(R^{+}, d\right)$ is a dislocated quasi-metric space, $f$ and $g$ satisfy the E. A. Like property and are weakly compatible.

$$
\begin{aligned}
& d(f x, f y)=d\left(\frac{1}{5} x, \frac{1}{5} y\right)=\frac{1}{5}(x+2 y) \\
& d(f x, g y)=d\left(\frac{1}{5} x, \frac{1}{4} y\right)=\frac{1}{5} x+\frac{1}{2} y \\
& d(g x, f y)=d\left(\frac{1}{4} x, \frac{1}{5} y\right)=\frac{1}{4} x+\frac{2}{5} y \\
& d(g x, g y)=d\left(\frac{1}{4} x, \frac{1}{4} y\right)=\frac{1}{4}(x+2 y) \\
& d(g y, f y)=d\left(\frac{1}{4} y, \frac{1}{5} y\right)=\frac{1}{4} y+\frac{2}{5} y \\
& d(g x, f x)=d\left(\frac{1}{4} x, \frac{1}{5} x\right)=\frac{1}{4} x+\frac{2}{5} x
\end{aligned}
$$

So,

$$
\begin{aligned}
& d(f x, f y)= \frac{1}{5}(x+2 y) \\
& \leq \frac{1}{24}\left(\frac{1}{5} x+\frac{1}{2} y\right)+\frac{1}{12}\left(\frac{1}{4} x+\frac{2}{5} y\right) \\
&+\frac{1}{4}\left(\frac{1}{4} x+\frac{1}{2} y\right)+\frac{1}{24}\left(\frac{1}{4} y+\frac{2}{5} y\right) \\
&+\frac{1}{24}\left(\frac{1}{4} x+\frac{2}{5} x\right) \\
&= \alpha d(f x, g y)+\beta d(g x, f y)+\gamma d(g x, g y) \\
&+\delta d(g y, f y)+\eta d(g x, f x) \\
& \text { for } \alpha=\delta=\eta= \frac{1}{24}, \beta=\frac{1}{12}, \gamma=\frac{1}{4} \\
& \text { and } 0 \leq \alpha+\beta+\gamma+\delta+\eta=\frac{11}{24}<\frac{1}{2} .
\end{aligned}
$$

All conditions of theorem 3.1 are satisfied. Thus 0 is the unique common fixed point of $f$ and $g$.

Theorem 3.3 Let $(X, d)$ be a dislocated quasi-metric space and $f, g: X \rightarrow X$ are self mappings, satisfying the conditions:

$$
\begin{aligned}
d(f x, f y) \leq & \alpha[d(f x, g y)+d(g x, f y)] \\
& +\beta[d(f x, g y)+d(g x, g y)] \\
& +\gamma[d(g x, f y)+d(g x, g y)]
\end{aligned}
$$

for all $x, y \in X$, and $0 \leq \alpha+\beta+\gamma<\frac{1}{4}$.

(3.3.2) $f$ and $g$ satisfy the E. A. Like property,

(3.3.3) $f$ and $g$ are weakly compatible

Then $f$ and $g$ have a unique common fixed point in $X$. Proof: Since $f$ and $g$ satisfy the E. A. Like Property therefore exists a sequence $\left(x_{n}\right)$ in $X$ such that $\lim _{n \rightarrow \infty} f x_{n}=\lim _{n \rightarrow \infty} g x_{n}=z \in f(X)$ or $g(X)$.

Assume that $\lim _{n \rightarrow \infty} f x_{n}=z \in g(X)$. Therefore, $z=g u$ for some $u \in X$.

From condition (3.3.1) we have:

$$
\begin{aligned}
d\left(f u, f x_{n}\right) \leq & \alpha\left[d\left(f u, g x_{n}\right)+d\left(g u, f x_{n}\right)\right] \\
& +\beta\left[d\left(f u, g x_{n}\right)+d\left(g u, g x_{n}\right)\right] \\
& +\gamma\left[d\left(g u, f x_{n}\right)+d\left(g u, g x_{n}\right)\right]
\end{aligned}
$$


Taking limit as $n \rightarrow \infty$, we get

$$
\begin{aligned}
d(f u, z) \leq & \alpha[d(f u, z)+d(z, z)] \\
& +\beta[d(f u, z)+d(z, z)] \\
& +\gamma[d(z, z)+d(z, z)] \\
& =(\alpha+\beta+2 \gamma) d(z, z)+(\alpha+\beta) d(f u, z) \\
& \leq(2 \alpha+2 \beta+2 \gamma) d(f u, z) \\
& +(\alpha+\beta+2 \gamma) d(z, f u)
\end{aligned}
$$

From this inequality have

$$
d(f u, z) \leq \frac{\alpha+\beta+2 \gamma}{1-(2 \alpha+2 \beta+2 \gamma)} d(z, f u) .
$$

In the same way from condition (3.3.1) have

$$
d(z, f u) \leq \frac{\alpha+2 \beta+\gamma}{1-(2 \alpha+2 \beta+2 \gamma)} d(f u, z) .
$$

By (3) and (4) we get $d(f u, z)=d(z, f u)=0$ since $0 \leq \alpha+\beta+\gamma<\frac{1}{4}$.

By property $d_{2}$ have $f u=z$. Hence $f u=g u=z$. Using the weak compatibility we get $f z=g z$.

Let we show that $f z=z$. Again consider:

$$
\begin{aligned}
d\left(f z, f x_{n}\right) \leq & \alpha\left[d\left(f z, g x_{n}\right)+d\left(g z, f x_{n}\right)\right] \\
& +\beta\left[d\left(f z, g x_{n}\right)+d\left(g z, g x_{n}\right)\right] \\
& +\gamma\left[d\left(g z, f x_{n}\right)+d\left(g z, g x_{n}\right)\right]
\end{aligned}
$$

Taking limit as $n \rightarrow \infty$, we get

$$
\begin{aligned}
d(f z, z) & \leq \alpha[d(f z, z)+d(f z, z)] \\
& +\beta[d(f z, z)+d(f z, z)] \\
& +\gamma[d(f z, z)+d(f z, z)] \\
& \leq(2 \alpha+2 \beta+2 \gamma) d(f z, z)
\end{aligned}
$$

From this have $d(f z, z)=0$ since $0 \leq \alpha+\beta+\gamma<\frac{1}{4}$, and again considering $d\left(f x_{n}, z\right)$ have $d(z, f z)=0$.

Therefore $\quad d(f z, z)=d(z, f z)=0 \Rightarrow f z=z$

So $f z=z=g z$. Hence, $z$ is a common fixed point of $f$ and $g$.

Uniqueness. Clearly, as in theorem 3.1 we can show that fixed point is unique.

Theorem 3.4 Let $(X, d)$ be a dislocated metric space and $S, T, F$ and $G$ are self mappings satisfying the conditions:

$$
\begin{aligned}
& d(S x, T y) \leq k_{1} d(F x, G y)+k_{2} d(F x, S x) \\
& +k_{3} d(G y, T y)+k_{4} d(F x, T y)+k_{5} d(G y, S x)
\end{aligned}
$$

for all $x, y \in X$, where the constants $k_{1}, k_{2}, k_{3}, k_{4}, k_{5}$ are nonnegative and $0 \leq k_{1}+k_{2}+k_{3}+k_{4}+k_{5}<\frac{1}{2}$.

(3.4.2) The pairs $(S, F)$ and $(T, G)$ satisfy common E. A. Like property,
(3.4.3) The pairs $(S, F)$ and $(T, G)$ are weakly compatible

Then $F, G, S$, and $T$ have a unique common fixed point in $X$.

Proof. Since $(S, F)$ and $(T, G)$ satisfy common E. A. Like property therefore there exists two sequences $\left(x_{n}\right)$ and $\left(y_{n}\right)$ in $X$ such that

$\lim _{n \rightarrow \infty} S x_{n}=\lim _{n \rightarrow \infty} F x_{n}=\lim _{n \rightarrow \infty} G y_{n}=\lim _{n \rightarrow \infty} T y_{n}=z$, where $z \in F(X) \cap G(X)$ or $z \in S(X) \cap T(X)$.

If we assume that $z \in F(X) \cap G(X)$, we have

$\lim _{n \rightarrow \infty} S x_{n}=z \in F(X)$ then $z=F u$ for some $u \in X$.

Now, claim that $S u=F u$, from condition (3.4.1) we have

$$
\begin{aligned}
& d\left(S u, T y_{n}\right) \leq k_{1} d\left(F u, G y_{n}\right)+k_{2} d(F u, S u) \\
& +k_{3} d\left(G y_{n}, T y_{n}\right)+k_{4} d\left(F u, T y_{n}\right)+k_{5} d\left(G y_{n}, S u\right)
\end{aligned}
$$

Taking limit $n \rightarrow \infty$, we get

$$
\begin{aligned}
d(S u, z) & \leq k_{1} d(z, z)+k_{2} d(z, S u)+k_{3} d(z, z) \\
& +k_{4} d(z, z)+k_{5} d(z, S u) \\
& \leq\left(2 k_{1}+k_{2}+2 k_{3}+2 k_{4}+k_{5}\right) d(S u, z)
\end{aligned}
$$

From this inequality and since $0 \leq k_{1}+k_{2}+k_{3}+k_{4}+k_{5}<\frac{1}{2} \quad$, get $\quad d(S u, z)=0 \quad$. Since $X$ is dislocated metric space, get $S u=z$. Hence $S u=F u=z(1)$.

Again $\lim _{n \rightarrow \infty} T y_{n}=z \in G(X)$, then $z=G v$ for some $v \in X$.

In the same way as above and using the same condition we show that $T v=G v=z$ (2). And by the weak compatibility of the pair $(S, F)$ obtain $S z=S F u=F S u=F z$.

Let we show now that $S z=z$.

$$
\begin{aligned}
& d\left(S z, T y_{n}\right) \leq k_{1} d\left(F z, G y_{n}\right)+k_{2} d(F z, S z) \\
& +k_{3} d\left(G y_{n}, T y_{n}\right)+k_{4} d\left(F z, T y_{n}\right)+k_{5} d\left(G y_{n}, S z\right)
\end{aligned}
$$

And taking limit $n \rightarrow \infty$, we get

$$
\begin{aligned}
d(S z, z) \leq & k_{1} d(S z, z)+k_{2} d(S z, S z)+k_{3} d(z, z) \\
& +k_{4} d(S z, z)+k_{5} d(z, S z) \\
& \left(k_{1}+2 k_{2}+2 k_{3}+k_{4}+2 k_{5}\right) d(S z, z)
\end{aligned}
$$

This implies $\quad d(S z, z)=0 \quad$ since $0 \leq k_{1}+k_{2}+k_{3}+k_{4}+k_{5}<\frac{1}{2}$. And since $X$ is dislocated metric space have $S z=z$, so $S z=F z=z$ (3).

From (2) and the weak compatibility of the pair $(T, G)$ get $G z=T z$ (4). By condition (3.4.1) consider:

$$
\begin{aligned}
& d\left(S x_{n}, T z\right) \leq k_{1} d\left(F x_{n}, G z\right)+k_{2} d\left(F x_{n}, S x_{n}\right) \\
& +k_{3} d(G z, T z)+k_{4} d\left(F x_{n}, T z\right)+k_{5} d\left(G z, S x_{n}\right)
\end{aligned}
$$

as $n \rightarrow \infty$,we get 


$$
\begin{aligned}
d(z, T z) \leq & k_{1} d(z, T z)+k_{2} d(z, z)+k_{3} d(T z, T z) \\
& +k_{4} d(z, T z)+k_{5} d(T z, z) \\
& \leq\left(k_{1}+2 k_{2}+2 k_{3}+k_{4}+k_{5}\right) d(z, T z)
\end{aligned}
$$

This implies $d(z, T z)=0$. Since $X$ is dislocated metric space and from (4) have $T z=z=G z$ (5).

Thus by (3) and (5) $z$ is a common fixed point of $S, T, F$ and $G$.

Uniqueness. Suppose that $z \neq z_{1}$ are two common fixed points of $S, T, F$ and $G$. Then from (3.4.1) we have:

$$
\begin{aligned}
d\left(z, z_{1}\right) & =d\left(S z, T z_{1}\right) \\
& \leq k_{1} d\left(F z, G z_{1}\right)+k_{2} d(F z, S z)+k_{3} d\left(G z_{1}, T z_{1}\right) \\
& +k_{4} d\left(F z, T z_{1}\right)+k_{5} d\left(G z_{1}, S z\right) \\
& =k_{1} d\left(z, z_{1}\right)+k_{2} d(z, z)+k_{3} d\left(z_{1}, z_{1}\right) \\
& +k_{4} d\left(z, z_{1}\right)+k_{5} d\left(z_{1}, z\right) \\
& \leq\left(k_{1}+2 k_{2}+2 k_{3}+k_{4}+k_{5}\right) d\left(z, z_{1}\right)
\end{aligned}
$$

which implies $d\left(z, z_{1}\right)=0 \quad$ since $0 \leq k_{1}+k_{2}+k_{3}+k_{4}+k_{5}<\frac{1}{2}$. Thus $z=z_{1}$ and $z$ is the unique common fixed point of $S, T, F$ and $G$.

Example 3.5 Let $X=[0,1]$ and $d(x, y)=x+y$ for all $x, y \in X$. Then the pair $(X, d)$ is a dislocated metric space. We define the functions $S, T, F$ and $G$ as follows:

$$
F x=\frac{x}{2}, G x=x, S x=\frac{x}{12}, T x=\frac{x}{6} .
$$

The pairs $(S, F)$ and $(T, G)$ satisfy common E. A. Like property and are weakly compatible. We have,

$$
\begin{aligned}
d(S x, T y)= & d\left(\frac{x}{12}, \frac{x}{6}\right)=\frac{1}{6}\left(\frac{x}{2}+y\right) \leq \frac{1}{4}\left(\frac{x}{2}+y\right) \\
= & \frac{1}{4} d(F x, G y) \\
\leq & k_{1} d(F x, G y)+k_{2} d(F x, S x)+k_{3} d(G y, T y) \\
& +k_{4} d(F x, T y)+k_{5} d(G y, S x)
\end{aligned}
$$

where the constants $k_{1}, k_{2}, k_{3}, k_{4}, k_{5}$ are nonnegative and $0 \leq k_{1}+k_{2}+k_{3}+k_{4}+k_{5}<\frac{1}{2}$.

Thus all conditions of theorem are satisfying and 0 is the unique common fixed point of $S, T, F$ and $G$.

Corollary 3.6 Let $(X, d)$ be a dislocated metric space and $T, F$ and $G$ are self mappings satisfying the conditions:

$$
\begin{aligned}
& d(T x, T y) \leq k_{1} d(F x, G y)+k_{2} d(F x, T x) \\
& +k_{3} d(G y, T y)+k_{4} d(F x, T y)+k_{5} d(G y, T x)
\end{aligned}
$$

for all $x, y \in X$, and $0 \leq k_{1}+k_{2}+k_{3}+k_{4}+k_{5}<\frac{1}{2}$.

(3.6.2) The pairs $(T, F)$ and $(T, G)$ satisfy common E.

A. Like property,

(3.6.3) The pairs $(T, F)$ and $(T, G)$ are weakly compatible
Then $F, G$ and $T$ have a unique common fixed point in $X$.

Proof. This theorem is taken as corollary of theorem 3.4 if we take in it $S=T$.

Corollary 3.7 Let $(X, d)$ be a dislocated metric space and $S, T, F$ and $G$ are self mappings satisfying the conditions:

(3.7.1) $d(S x, T y) \leq k\left[\begin{array}{l}d(F x, G y)+d(F x, S x) \\ +d(G y, T y)+d(F x, T y)+d(G y, S x)\end{array}\right]$

for all $x, y \in X$, and $0 \leq k<\frac{1}{10}$.

(3.7.2) The pairs $(S, F)$ and $(T, G)$ satisfy common E. A. Like property,

(3.7.3) The pairs $(S, F)$ and $(T, G)$ are weakly compatible

Then $F, G, S$, and $T$ have a unique common fixed point in $X$.

Proof. If in theorem 3.4 we take $k_{1}=k_{2}=k_{3}=k_{4}=k_{5}$ we obtain above corollary (in a Lipschitz form contraction)

Corollary 3.8 Let $(X, d)$ be a dislocated metric space and $S, T, F$ and $G$ are self mappings satisfying the condition $_{\mathrm{S} \text { : }}$

$$
\begin{aligned}
d(S x, T y) \leq & \alpha d(F x, G y)+\beta[d(F x, S x)+d(G y, T y)] \\
& +\gamma[d(F x, T y)+d(G y, S x)]
\end{aligned}
$$

for all $x, y \in X$, and $0 \leq \alpha+\beta+\gamma<\frac{1}{2}$.

(3.8.2) The pairs $(S, F)$ and $(T, G)$ satisfy common E. A. Like property,

(3.8.3) The pairs $(S, F)$ and $(T, G)$ are weakly compatible

Then $F, G, S$, and $T$ have a unique common fixed point in $X$.

Proof. If in theorem 3.4 replace: $k_{1}=\alpha, k_{2}=k_{3}=\beta, k_{4}=k_{5}=\gamma$ we obtain this corollary

\section{References}

[1] Aamri, M. and El Moutawakil, D. Some new common fixed point theorems under strict contractive conditions, J. Math. Anal. Appl. 270, 181-188, 2002.

[2] C. T. Aage and J. N. Salunke. The results on fixed points in dislocated and dislocated quasi-metric space. Appl. Math. Sci.,2(59):2941-2948, 2008.

[3] F. M. Zeyada, G. H. Hassan, and M. A. Ahmed. A generalization of a fixed point theorem due to Hitzler and Seda in dislocated quasi-metric spaces. The Arabian J. for Sci. and Eng., 31(1A): 111:114, 2005.

[4] G. Jungck and B.E. Rhoades, Fixed points For Set Valued Functions without Continuity, Indian J. Pure Appl. Math., 29 (3) (1998), 227-238.

[5] Liu, W., Wu, J. and Li, Z. Common fixed points of single-valued and multi-valued maps, Int.J. Math. Math. Sc. 19, 3045-3055, 2005 .

[6] K. Zoto, Weakly compatible mappings and fixed points in dislocated -metric spaces, International journal of mathematical archive, vol. 4 (6), 2013, 131-137.

[7] K. Zoto and E. Hoxha, Fixed point theorems in dislocated and dislocated quasi-metric spaces, Journal of Advanced Studies in Topology; Vol. 3, No.4, 2012. 
[8] K. Jha and D. Panthi, A Common Fixed Point Theorem in Dislocated Metric Space, Appl. Math. Sci., vol. 6, 2012, no. 91, 4497-4503.

[9] K. P. R. Rao and P. Rangaswamy, Common Fixed Point Theorem for Four Mappings in Dislocated Quasi-Metric Space, The Nepali Math. Sci. Report, 30 (1-2), 2010, 70-75.

[10] P. Hitzler and A. K. Seda. Dislocated topologies. J. Electr. Engin., 51(12/S):3:7, 2000

[11] P. S Kumari, Common fixed point theorems on weakly compatible maps on dislocated metric spaces, Mathematical Sciences 2012, 6:71.

[12] R.Shrivastava, Z.K.Ansari and M.Sharma. Some results on Fixed Points in Dislocated and Dislocated Quasi-Metric Spaces. Journal of Advanced Studies in Topology; Vol. 3, No.1, 2012.

[13] S. K. Vats, Weakly Compatible Maps in Metric Spaces, J. Indian Math. Soc., 69 (1-4), (2002), 139-143.

[14] M. Arshad, A. Shoaib and P. Vetro; Common fixed points of a pair of Hardy Rogers type mappings on a closed ball in ordered dislocated metric spaces. Journal of function spaces and applications, vol 2013, article id 638181.
[15] E. Karapinar and P. Salimi, Dislocated metric space to metric-like spaces with fixed point theorems. Fixed Point Theory and Applications 2013, 2013: 222.

[16] M. Arshad, A. Shoaib and I. Beg, Fixed point of a pair of contractive dominated mappings on a closed ball in an ordered dislocated metric space. Fixed Point Theory and Applications 2013.

[17] Yijie Ren,Junlei Li, and Yanrong Yu, Common fixed point theorems for nonlinear contractive mappings in dislocated metric spaces. Abstract and Applied Analysis vol 2013, article id 483059.

[18] N. Hussain, J.R. Roshan, V. Parvaneh and M. Abbas; Common fixed point results for weak contractive mappings in ordered $b$ dislocated metric spaces with applications. Journal of Inequalities and Applications 2013, 2013:486.

[19] P Sumati Kumariet al, New Version for Hardy and Rogers Type Mapping in Dislocated Metric Space. International Journal of Basic and Applied Sciences, 1 (4) (2012) 609-617.

[20] K. Jha, D. Panthi; A common Fixed Point Theorem In Dislocated Metric Space, Applied Mathematical Sciences 2012.

[21] K. Wadhwa, H. Dubey, R. Jain; Impact of E. A. Like property on common fixed point theorems in fuzzy metric spaces.J. Adv. Stud. Topology 3 (1) (2012), 52-59. 